\title{
INFINITESIMAL CHARACTERIZATION OF HOMOGENEOUS BUNDLES
}

\author{
KIRILL MACKENZIE
}

(Communicated by David G. Ebin)

\begin{abstract}
Consider a principal bundle $Q(B, H)$ on a base $B$ which is compact and has finite fundamental group. We give necessary and sufficient conditions, in terms of the Atiyah sequence of $Q(B, H)$, for $Q(B, H)$ to be locally isomorphic to a bundle of the form $G(G / S, S)$ for $G$ a Lie group and $S$ a closed subgroup of $G$. The proof involves the careful integration of certain infinitesimal actions of a Lie algebra on $Q, B$ and the universal cover of $B$.
\end{abstract}

By a homogeneous bundle we mean a principal bundle of the form $G(G / H, H)$, where $G$ is a Lie group and $H$ is a closed subgroup. The infinitesimal properties of an arbitrary principal bundle $Q(B, H, p)$ are encoded in its Atiyah sequence

$$
\frac{Q \times \mathfrak{h}}{H} \succ \frac{T Q}{H} \stackrel{p_{*}}{\rightarrow} T B
$$

in which $T Q / H$ is the vector bundle on $B$ quotiented from $T Q$ by the action $X h=T\left(R_{h}\right)(X), p_{*}$ is the quotient of $T(p): T Q \rightarrow T B$, and $(Q \times \mathfrak{h}) / H$ is the Lie algebra bundle associated to $Q(B, H)$ by the adjoint representation. [5] gives a detailed account of the Atiyah sequence and its properties and, in particular, shows that all of the infinitesimal connection theory of $Q(B, H)$ takes place in the Atiyah sequence, rather than in the bundle itself. Further, the Atiyah sequence of a principal bundle behaves much like the Lie algebra of a Lie group: a morphism of Atiyah sequences integrates to a local morphism of principal bundles; a principal bundle $Q(B, H)$ with $Q$ connected has a universal covering (or monodromy) bundle $\widetilde{Q}(B, \widehat{H})$, where $\widetilde{Q}$ is the universal cover and $\widehat{H}$ is locally isomorphic to $H$, and the natural map $\widetilde{Q}(B, \widehat{H}) \rightarrow Q(B, H)$ induces an isomorphism of Atiyah sequences; there is a correspondence between reductions of a principal bundle and suitable subobjects of its Atiyah sequence. See [5] for an account of this theory and historical references.

The purpose of this paper is to give a partial answer to the following question, put to the author by E. Ruh in 1986: Given the Atiyah sequence $(*)$ of a principal bundle $Q(B, H)$, what properties of the Atiyah sequence ensure that the bundle is homogeneous? We answer this question provided that $B$ is compact and has finite fundamental group, showing that it is then necessary and sufficient that $T Q / H$ be trivializable as a vector bundle in such a way that the bracket of constant sections is constant, and that the constant vector fields on $Q$ commute with the fundamental vector fields. The result establishes that the monodromy bundle $\widetilde{Q}(B, \widehat{H})$ must be a homogeneous bundle; $Q(B, H)$ itself may be a quotient of this by a discrete

Received by the editors July 20, 1987.

1980 Mathematics Subject Classification (1985 Revision). Primary 55R91; Secondary 53C30. 
normal subgroup of $\widehat{H}$. The proof relies on the integration of infinitesimal actions of Lie groups; it is at this point that the compactness assumptions are used.

It is important for our purposes that a principal bundle isomorphic to a homogeneous bundle should be homogeneous. We therefore have to broaden the definition slightly: a homogeneous bundle is a principal bundle $Q(B, H, p)$, together with a Lie group $G$ acting transitively to the left on $Q$ and $B$, such that $p$ is equivariant, the actions of $G$ and $H$ on $Q$ commute, and the action of $G$ on $Q$ is free, as well as transitive. In particular a homogeneous bundle is equivariant in the sense of [4].

The first section briefly describes the Atiyah sequence of a homogeneous bundle, the second gives the main result in the case of a simply connected base manifold, and the third gives the general result.

I am most grateful to E. Ruh for putting the original question, and to $\mathrm{A}$. Weinstein for the crucial formula in 1.2. I also thank P. J. Higgins for some valuable comments.

1. The Atiyah sequence of a homogenous bundle. Consider a principal bundle $Q(B, H, p)$. Then $H$ acts on the tangent bundle $T Q \rightarrow Q$ by $X h=$ $T\left(R_{h}\right)(X)$, where $R_{h}: Q \rightarrow Q$ is the right translation corresponding to $h$, and the quotient is a vector bundle $T Q / H \rightarrow B$. We denote elements of $T Q / H$ by $\langle X\rangle$, where $X \in T Q$. Define $p_{*}: T Q / H \rightarrow T B$ by $p_{*}(\langle X\rangle)=T(p)(X)$; then $p_{*}$ is a surjective submersion of vector bundles over $B$. For $V \in \mathfrak{h}$, let $V^{*}$ denote the fundamental vector field $V^{*}(u)=T(h \mapsto u h)_{1}(V)$ on $Q$. Lastly, quotient the trivial bundle $Q \times \mathfrak{h} \rightarrow Q$ over the action of $H,(u, V) h=\left(u h, A d h^{-1} V\right)$; the result is a vector bundle $(Q \times \mathfrak{h}) / H \rightarrow B$, whose elements we denote by $\langle u, V\rangle$, and the map $(Q \times \mathfrak{h}) / H \rightarrow T Q / H,\langle u, V\rangle \mapsto\left\langle V^{*}(u)\right\rangle$ is an injective vector bundle morphism over $B$ making

$$
\frac{Q \times \mathfrak{h}}{H} \longmapsto \frac{T Q}{H} \stackrel{p_{*}}{\rightarrow} T B
$$

an exact sequence of vector bundles.

Sections of $T Q / H$ are in bijective correspondence with $H$-invariant vector fields on $Q$. Since the bracket of invariant vector fields is invariant, $\Gamma(T Q / H)$ acquires a bracket [,] which is alternating, obeys the Jacobi identity, and has the properties

$$
\begin{gathered}
{[X, f Y]=f[X, Y]+p_{*}(X)(f) Y} \\
p_{*}([X, Y])=\left[p_{*}(X), p_{*}(Y)\right]
\end{gathered}
$$

for $X, Y \in \Gamma(T Q / H), f \in C(B)$. Here $C(B)$ is the ring of smooth functions on $B$.

From (3) it follows that the bracket on $\Gamma(T Q / H)$ restricts to $\Gamma((Q \times \mathfrak{h}) / H)$. In terms of $H$-equivariant maps $Q \rightarrow \mathfrak{h}$ this bracket is

$$
[V, W](u)=[V(u), W(u)]_{R},
$$

where $[,]_{R}$ is the bracket on $\mathfrak{h}$ induced from the right-invariant vector fields on $H$. In particular, $(Q \times \mathfrak{h}) / H$ is a Lie algebra bundle.

A full account of this construction, with references, is given in [5, Appendix A]. We will need the following example shortly.

EXAMPLE 1.1. Consider a trivial bundle $B \times H(B, H)$. Then $T(B \times H) / H \cong$ $T B \oplus(B \times \mathfrak{h})$, where $\oplus$ is the direct sum over $B$, and $(B \times H \times \mathfrak{h}) / H$ is isomorphic 
to the trivial Lie algebra bundle $B \times \mathfrak{h}$. The bracket on $T B \oplus(B \times \mathfrak{h})$ is

$$
[X \oplus V, Y \oplus W]=[X, Y] \oplus\left\{X(W)-Y(V)+[V, W]_{R}\right\}
$$

where $X(W)$ and $Y(V)$ denote Lie derivatives. Compare [5, III 3.21].

Now consider a bundle of the form $G(G / H, H, p)$, where $G$ is a Lie group and $H$ is a closed subgroup. Using the right trivialization of $T G$, one easily sees that $T G / H$ has a trivialization

$$
\langle X\rangle \mapsto\left(g H, T\left(R_{g}-1\right)(X)\right), \quad \frac{T G}{H} \rightarrow(G / H) \times \mathfrak{g}
$$

where $X \in T(G)_{g}$. The map $p_{*}$ now becomes

$$
(G / H) \times \mathfrak{g} \rightarrow T(G / H), \quad(x, X) \mapsto \bar{X}(x)
$$

where $\bar{X}(x)=T(g \mapsto g x)_{1}(X)$ is the vector field on $G / H$ induced by $X$ via the action of $G$ on $G / H$.

The following formula is due to A. Weinstein.

Proposition 1.2. The bracket on $\Gamma(T G / H)$ is given in term of maps $G / H \rightarrow$ $\mathfrak{g} b y$

$$
[X, Y]=\bar{X}(Y)-\bar{Y}(X)+[X, Y]^{\circ}
$$

where $X, Y: G / H \rightarrow \mathfrak{g}$. Here [, ]' is the pointwise (right-hand) bracket and $\bar{X}$ is the vector field on $G / H$ defined by $\bar{X}(x)=\overline{(X(x))}(x)$; that is, $\bar{X}(x)=$ $T(g \mapsto g x)_{1}(X(x))$.

ProOF. Consider the morphism $\varphi$ from $G(G / H, H)$ to the trivial bundle $(G / H)$ $\times G(G / H, G)$ defined by $\varphi(g)=(g H, g)$. Because this is a morphism of principal bundles, it induces a morphism of their Atiyah sequences $\varphi_{*}:(G / H) \times \mathfrak{g} \rightarrow$ $T(G / H) \oplus(G / H \times \mathfrak{g})$. (See, for example [5, A§3].) Now because $\varphi$ induces the identity map $G / H \rightarrow G / H$, the first component of $\varphi_{*}(x, X)$ must be $\bar{X}(x)$, and it is easy to see that the second is $(x, X)$ itself. Briefly, we write $\varphi_{*}(X)=\bar{X} \oplus X$. Now apply the formula from 1.1 .

It is clear from 1.2 that the bracket of constant sections is constant. Indeed the constant sections of $T Q / H$ corresponding to this trivialization are those of the form $g H \mapsto\left\langle T\left(R_{g}\right)_{1}(X)\right\rangle$, where $X$ is a fixed element of $\mathfrak{g}$. These in turn correspond to the right-invariant vector fields on $G$. Since the fundamental vector fields on $G$ are precisely those left-invariant vector fields which correspond to elements of $\mathfrak{h} \leq \mathfrak{g}$, it is immediate that the "constant" fields (in the sense of 2.1) on $G$ commute with the fundamental vector fields.

2. The case of a simply-connected base manifold. Given a principal bundle $Q(B, H)$ with $Q$ disconnected one may choose any component $Q_{0}$ and obtain a reduction $Q_{0}\left(B, H^{\prime}\right)$, where $H^{\prime}=\left\{h \in H \mid Q_{0} h=Q_{0}\right\}$, and it is immediate that the inclusion $Q_{0}\left(B, H^{\prime}\right) \longmapsto Q(B, H)$ induces an isomorphism of Atiyah sequences. Further, for any $Q(B, H)$ with $Q$ connected there is a natural monodromy bundle $\widetilde{Q}(B, \widehat{H})$, where $\widetilde{Q}$ is the universal covering of $Q$, and $\widehat{H}$ is a certain extension $\pi_{1} Q \mapsto \widehat{H} \rightarrow H$, such that the covering projection $\widetilde{Q} \rightarrow Q$ is a morphism of principal bundles $\widetilde{Q}(B, \widehat{H}) \rightarrow Q(B, H)$ and induces an isomorphism of Atiyah sequences. 
(See, for example, [5, II $\S 6$, III $\S 6]$.) It therefore follows that we may suppose that we are given the Atiyah sequence

$$
\frac{Q \times \mathfrak{h}}{H} \nrightarrow \frac{T Q}{H} \rightarrow T B
$$

of a bundle $Q(B, H)$ in which $Q$ is simply-connected.

Notice too that if $G$ is a simply-connected Lie group, $H$ a closed subgroup of $G$ and $D$ a discrete normal subgroup of $H$, which is not normal in $G$, then $G(G / H, H)$ and $G / D(G / H, H / D)$ will have the same Atiyah sequence, but only the first will be a homogeneous bundle. The bundles $S U(2)\left(S^{2}, U(1)\right)$ and $S U(2) / \mathbf{Z}_{n}\left(S^{2}, U(1) / \mathbf{Z}_{n}\right)$ for $n>2$ are examples. On the other hand, the monodromy bundle of a homogeneous bundle is homogeneous [5, II 6.5].

We now come to the main result.

THEOREM 2.1. Let $Q(B, H, p)$ be a principal bundle with $Q$ simply-connected and $B$ compact and simply-connected. Suppose that $T Q / H$ is trivializable as a vector bundle in such a way that (i) the bracket of constant sections of $T Q / H$ is constant, and (ii) the constant vector fields on $Q$ (that is, the $H$-invariant vector fields corresponding to the constant sections of $T Q / H)$ commute with the fundamental vector fields. Then $Q(B, H)$ is a homogeneous bundle.

PrOOF. Let the trivialization be $\varphi: T Q / H \cong B \times V$. Because the bracket of constant sections is constant, $V$ acquires a Lie algebra structure and we henceforth denote it by $\mathfrak{g}$. (Notice that the bracket of any two maps $B \rightarrow \mathfrak{g}$ is now determined by condition (2) of $\S 1$.)

Now $\varphi$ lifts to another isomorphism of vector bundles, $\Phi: T Q \rightarrow Q \times \mathfrak{g}$, which is $H$-equivariant (where $H$ acts on $Q \times \mathfrak{g}$ by $(u, X) h=(u h, X))$. Given $X \in \mathfrak{g}$, the corresponding constant vector field, denoted $\vec{X}$, is $\vec{X}(u)=\Phi^{-1}(u, X)$; it is $H$-invariant. A general result of Kumpera $[3, \S 33]$ asserts that an invariant vector field on a principal bundle is complete iff its projection to the base is complete; since $B$ is here assumed compact, it follows that $\vec{X}$ is complete.

From well-known results of Palais, in the form given in $[2,3.1 .3]$, we obtain an action $G \times Q \rightarrow Q$ with the property that

$$
T(g \mapsto g u)_{1}(X)=\vec{X}(u)
$$

for all $u \in Q, X \in \mathfrak{g}$, where $G$ is the simply-connected Lie group corresponding to $\mathfrak{g}$. Also, because $\Phi$ is injective, it follows that each $\mathfrak{g} \rightarrow T(Q)_{u}, X \mapsto \vec{X}(u)$, is injective, and therefore the action of $G$ is locally free. Lastly, each $\mathfrak{g} \rightarrow T(Q)_{u}$ is an isomorphism, and so the orbits of $G$ are open in $Q$; since $Q$ is connected it follows that the action is transitive. So we have a transitive, locally free action, and therefore each evaluation map $G \rightarrow Q, g \mapsto g u$ is a covering; since $Q$ is simplyconnected, each evaluation map must be a diffeomorphism. Thus $G \times Q \rightarrow Q$ is a simply transitive action.

Next define $\mathfrak{g} \rightarrow \Gamma T B, X \mapsto \bar{X}$, by $\bar{X}(x)=\left(p_{*} \circ \varphi^{-1}\right)(x, X)$. Again, each $\bar{X}$ is complete, and by integration we obtain an action $G \times B \rightarrow B$, with

$$
T(g \mapsto g x)_{1}(X)=\bar{X}(x)
$$


for $x \in B, X \in \mathfrak{g}$. Since $p_{*}$ is a surjective submersion, each map $\mathfrak{g} \rightarrow T(B)_{x}$, $X \mapsto \bar{X}(x)$ is surjective and so, as before, the action of $G$ is transitive. Lastly, $\bar{X}$ is the projection under $p: Q \rightarrow B$ of the $H$-invariant vector field $\vec{X}$ and it follows that $p$ is $G$-equivariant.

Return now to $Q$. We have a left $G$-action and a right $H$-action and wish to show that they commute. By assumption, $\left[\vec{X}, V^{*}\right]=0$ for all $X \in \mathfrak{g}, V \in \mathfrak{h}$. Now the action of $G$ on $Q$ provides $\vec{X}$ with a global flow, namely $\theta_{t}(u)=\exp t X u$. From general considerations, $V^{*}$ has the global flow $\psi_{s}(u)=u \exp s V$. Commutativity of $\vec{X}$ and $V^{*}$ implies that $\theta_{t} \circ \psi_{s}=\psi_{s} \circ \theta_{t}$ for all $s, t \in \mathbf{R}$ and therefore

$$
\exp t X(u \exp s V)=(\exp t X u) \exp s V
$$

for all $u \in Q$. Now $G$ and $H$ are both connected, $G$ by assumption and $H$ by putting $\pi_{0} Q=\pi_{1} B=0$ in the homotopy sequence of $Q(B, H)$. So $\exp (\mathfrak{g})$ generates $G$ and $\exp (\mathfrak{h})$ generates $H$ and the above equation now implies that $g(u h)=(g u) h$ for all $g \in G, u \in Q, h \in H$.

This completes the proof.

If one wants a bundle of the form $G(G / S, S)$, where $S$ is a subgroup of $G$ and $G(G / S, S) \cong Q(B, H)$, one can proceed as follows. Fix $u_{0} \in Q$ and $x_{0}=p\left(u_{0}\right) \in B$. Let $S$ be the stability subgroup of $G$ at $x_{0}$. Then each $s \in S$ sends $u_{0}$ to $s u_{0} \in$ $p^{-1}\left(x_{0}\right)$ and so there exists a unique $h \in H$ with $s u_{0}=u_{0} h^{-1}$. Write $h=f(s)$. Because the $G$ and $H$ actions commute, it follows that $f: S \rightarrow H$ is a morphism, and it is easily seen to be an isomorphism. We now have a trio of maps $F: G \rightarrow Q$, $g \mapsto g u_{0} ; f: S \rightarrow H ; F_{0}: G / S \rightarrow B, g S \mapsto g x_{0}$. It is easily checked that they give an equivariant isomorphism of principal bundles $F\left(F_{0}, f\right): G(G / S, S) \rightarrow Q(B, H)$, using again the commutativity of the $G$ and $H$ actions. The smoothness of $f$ follows from that of $F$.

Concerning the topological assumptions on $Q$ and $B$, we already noted that the simple-connectivity of $Q$ is merely a procedural hypothesis. The condition that $B$ be simply-connected was introduced only to force $H$ to be connected, and we remove this in $\S 3$. However the requirement that $B$ be compact is essential to the method of proof, and we see no way of doing without it.

3. Multiply-connected base manifolds. The idea here is to lift the bundle to the universal cover of the base, and to show that the resulting homogeneous bundle induces a homogeneous bundle on the original base. We assume that we are given a principal bundle $Q(B, H, p)$, with $Q$ simply-connected, with $B$ and the universal cover $\widetilde{B}$ compact, and whose Atiyah sequence satisfies the conditions (i) and (ii) of 2.1. Denote the covering projection $\widetilde{B} \rightarrow B$ by $c$ and $\pi_{1} B$ by $\pi$.

Lift $p: Q \rightarrow B$ to $\widetilde{p}: Q \rightarrow \widetilde{B}$. Explicitly, one can fix $u_{0} \in Q$, and for any $u \in Q$, take a path in $Q$ from $u_{0}$ to $u$ and let $\widetilde{p}(u)$ be the homotopy class of the projection of this path under $p$. Writing $x_{0}=p\left(u_{0}\right)$, one has $\widetilde{p}\left(u_{0}\right)=\widetilde{x}_{0}$, the class of the path in $B$ constant at $x_{0}$. Now $\widetilde{p}: Q \rightarrow \widetilde{B}$ is a principal bundle with group $H_{0}$, the identity component of $H$, and is a reduction of the inverse-image bundle $c^{*} Q(\widetilde{B}, H)$. Indeed one can express $c^{*} Q(\widetilde{B}, H)$ as $Q \times \pi(\widetilde{B}, H)$. Here the projection $Q \times \pi \rightarrow \widetilde{B}$ is $(u, \lambda) \mapsto \widetilde{p}(u) \lambda$ and the action of $H$ is $(u, \lambda) h=\left(u h, \partial(h)^{-1} \lambda\right)$, where $\partial$ is the natural morphism $H \rightarrow \pi_{0} H \cong \pi$. 
For the Atiyah sequence of $Q\left(\widetilde{B}, H_{0}\right)$, one sees firstly that as vector bundles there are isomorphisms

$$
\begin{array}{ccccc}
\frac{Q \times \mathfrak{h}}{H_{0}} & \longmapsto & \frac{T Q}{H_{0}} & \stackrel{p_{*}}{\rightarrow} & T \widetilde{B} \\
\text { 2॥ } & & \text { ॥ } & & \text { 2॥ } \\
c^{*}\left(\frac{Q \times \mathfrak{h}}{H}\right) & \mapsto & c^{*}\left(\frac{T Q}{H}\right) & \rightarrow & c^{*}(T B)
\end{array}
$$

Now the module of sections of an inverse-image bundle can be represented as a tensor product, $\Gamma\left(c^{*}(T Q / H)\right) \cong C(\widetilde{B}) \otimes_{C(B)} \Gamma(T Q / H)$. Here $C(\widetilde{B})$ and $C(B)$ are the rings of smooth functions and $\varphi \otimes X$ corresponds to $\varphi(X \circ c)$. In these terms, $p_{*}$ becomes $\varphi \otimes X \rightarrow \varphi \otimes p_{*}(X)$, regarding $\Gamma\left(c^{*}(T B)\right)$ as $C(\widetilde{B}) \otimes_{C(B)} \Gamma T B$, and the bracket becomes

$$
[\varphi \otimes X, \psi \otimes Y]=\varphi \psi \otimes[X, Y]+\varphi \bar{X}(\psi) \otimes Y-\psi \bar{Y}(\varphi) \otimes X
$$

where $\bar{X}, \bar{Y}$ denote the $\pi$-invariant vector fields on $\widetilde{B}$ which correspond to $p_{*}(X)$, $p_{*}(Y) \in \Gamma T B \cong \Gamma^{\pi} T \widetilde{B}$. This formula is forced by equation (2) of $\S 1$.

It is clear that $T Q / H_{0} \cong c^{*}(T Q / H) \cong \widetilde{B} \times \mathfrak{g}$. Further, the constant sections of $c^{*}(T Q / H)$ are precisely those of the form $1 \otimes X$, where $X$ is a constant section of $T Q / H$ and 1 is the function constant at 1 . The bracket formula above now shows that it is still true that the bracket of constant sections is constant. Lastly, the constant vector fields on $Q$ arising from $T Q / H_{0} \cong \widetilde{B} \times \mathfrak{g}$ are identical to the constant vector fields for $T Q / H \cong B \times \mathfrak{g}$, and the fundamental vector fields are also the same. Thus all the conditions of 2.1 apply to $Q\left(\widetilde{B}, H_{0}, \widetilde{p}\right)$ and we obtain $Q\left(\widetilde{B}, H_{0}\right) \cong G(G / S, S)$ where $G$ acts transtively on $\widetilde{B}$ with stability subgroup $S$ at $x_{0}$.

We now want to show that the actions of $G$ and $\pi$ on $\widetilde{B}$ commute. To do this, consider the action of $G$ on $B$ induced by $T Q / H \cong B \times \mathfrak{g}$. As before, this action is transitive and makes $c$ equivariant. Because $G$ is simply-connected, there is a canonical lift of the action of $G$ on $B$ to $\widetilde{B}$; by uniqueness this must be the same action as obtained from $T Q / H_{0} \cong \widetilde{B} \times \mathfrak{g}[\mathbf{1}$, I.9]. Now the canonical lift action certainly commutes with the action of $\pi$.

We now have bundles $Q\left(\widetilde{B}, H_{0}, \tilde{p}\right)$ and $\widetilde{B}(B, \pi, c)$ and we want to show that the composite $c \circ \widetilde{p}: Q \rightarrow B$ is a homogeneous bundle. Since $Q\left(\widetilde{B}, H_{0}\right)$ is equivariantly isomorphic to a $G(G / S, S)$, we can work directly with the latter. We now have the homogeneous bundle $G(G / S, S, \widetilde{p})$ and a bundle $G / S(B, \pi, c)$, where $G$ and $\pi$ commute on $G / S, G$ acts transitively on $B$, and $c$ is equivariant. We have basepoints $1 \in G, S \in G / S$, and $x_{0} \in B$ with $\tilde{p}(1)=S$ and $c(S)=x_{0}$. Denote the action of $\pi$ on $G / S$ by $(g S, \lambda) \mapsto(g S) \lambda$.

Define $K=(c \circ \widetilde{p})^{-1}\left(x_{0}\right) \subset G$. Then $K$ is a subgroup of $G$. For example, if $k_{1}, k_{2} \in K$ then $k_{1} S=S \lambda_{1}, k_{2} S=S \lambda_{2}$ for some $\lambda_{1}, \lambda_{2} \in \pi$, and $k_{1} k_{2} S=$ $k_{1}\left(S \lambda_{2}\right)=\left(k_{1} S\right) \lambda_{2}=S \lambda_{1} \lambda_{2}$, so $k_{1} k_{2} \in K$. Lastly the cosets $g K, g \in G$, are identical to the fibres of $c \circ \tilde{p}$ : for $g \in G, k \in K$ we have

$$
\begin{aligned}
(c \circ \widetilde{p})(g k) & =c(g(k S))=c(g(S \lambda)) \quad(\text { for some } \lambda \in \pi) \\
& =c((g S) \lambda)=(c \circ \widetilde{p})(g),
\end{aligned}
$$


and if $g_{1}, g_{2} \in G$ and $g_{1} S=\left(g_{2} S\right) \lambda$ for some $\lambda \in \pi$, then $g_{1} S=g_{2}(S \lambda)=g_{2}(k S)$ for some $k \in K$, and hence $g_{1}=g_{2} k s$. It is clear that $S \subset K$. The same calculations in fact prove that $K$ is the stability subgroup of $G$ acting on $B$ with reference point $x_{0}$.

We therefore have that $c \circ \tilde{p}: G \rightarrow B$ is (isomorphic to) a homogeneous bundle $G(G / K, K)$. This last part of the proof could be paraphrased as saying that the composite of a homogeneous bundle with an equivariant bundle is a homogeneous bundle.

Summarizing we have the following result.

THEOREM 3.1. Let $Q(B, H, p)$ be a principal bundle with $Q$ simply-connected and $B$ compact with finite fundamental group. Suppose that $T Q / H$ is trivializable as a vector bundle in such a way that the conditions (i) and (ii) of 2.1 are satisfied. Then $Q(B, H)$ is a homogeneous bundle.

Notice that in 2.1 we actually proved slightly more than we have here. In 2.1 the bundle $Q(B, H)$ acquired an action from $G$ which made it an equivariant bundle, and the isomorphism $G(G / S, S) \rightarrow Q(B, H)$ was an isomorphism of equivariant bundles. Here the actions of $G$ on $Q$ and $B$ need not make $Q(B, H)$ an equivariant bundle; all we assert is that there is a bundle isomorphism $G(G / S, S) \rightarrow Q(B, H)$.

It would be interesting to know whether (i) and (ii) are sufficient when the base is not compact.

\section{REFERENCES}

1. G. Bredon, Introduction to compact transformation groups, Academic Press, New York and London, 1972.

2. G. Hector and U. Hirsch, Introduction to the geometry of foliations, Part A, 2nd ed., Vieweg and Sohn, Braunschweig and Wiesbaden, 1986.

3. A. Kumpera and D. C. Spencer, Lie equations, vol. I, Princeton Univ. Press, Princeton, N.J., 1972.

4. R. K. Lashof, J. P. May and G. B. Segal, Equivariant bundles with abelian structural group, Contemp. Math. vol. 19, Amer. Math. Soc., Providence, R.I., 1983.

5. K. Mackenzie, Lie groupoids and Lie algebroids in differential geometry, London Math. Soc. Lecture Notes Series, no. 124, Cambridge Univ. Press, 1987.

Department of Mathematical SCIENCEs, University of DuRham, SCIENCE LABORATORIES, SOUTH ROAD, DURHAM DH1 3LE, ENGLAND 